\title{
Enucleación esofágica de leiomioma asistida por robot: reporte de un caso y revisión de la literatura
}

\section{Case report of robot-assisted esophageal enucleation of leiomyoma and literature review}

Miguel Ricardo Buitrago Ramírez, MD, ${ }^{1}$ Jorge Ramírez Pereira, MD, ${ }^{2}$ Diego Fernando Salcedo Miranda, MD. ${ }^{*}$

\footnotetext{
1 Cirujano de tórax, Clínica Marly y Universidad El Bosque. Bogotá D. C., Colombia.

2 Fellow de cirugía de tórax, Universidad El Bosque. Bogotá D. C., Colombia.

3 Residente de cirugía general, Universidad El Bosque. Bogotá D. C., Colombia.

*Correspondencia: dsalcedo_ub@hotmail.com.
}

Fecha recibido: $23 / 10 / 17$

Fecha aceptado: 04/03/18

\begin{abstract}
Resumen
Introducción: los leiomiomas esofágicos son tumores benignos, que son resecados por enucleación esofágica cuando son mayores de $5 \mathrm{~cm}$ o en lesiones de cualquier tamaño que sean sintomáticos o sospechosos de malignidad. Tradicionalmente, se ha realizado la resección por técnica de enucleación abierta; sin embargo, la cirugía mínimamente invasiva ha surgido como una técnica con grandes ventajas y, en especial, la tecnología robótica parece ofrecer ventajas. Caso clínico: se reporta un caso de leiomioma esofágico del esófago medio con enucleación mediante el uso de una técnica toracoscópica asistida por robot. La esofagoscopia intraoperatoria y la transiluminación fueron complementos útiles para identificar el esófago y desarrollar un plano de disección extramucosa seguro, que junto con la asistencia robótica parecen minimizar los riesgos intraoperatorios, entre ellos, potencialmente la probabilidad de lesión mucosa, y mejoran el tiempo de recuperación postoperatoria.
\end{abstract}

Palabras clave

Leiomioma esofágico, tumor benigno, robótica, enucleación, cirugía mínimamente invasiva.

\begin{abstract}
Introduction: Esophageal leiomyomas are benign tumors which are resected by esophageal enucleation when they are symptomatic, suspected of malignancy, or larger than $5 \mathrm{~cm}$. Traditional resection uses the open enucleation technique, but minimally invasive surgery has emerged as a technique that has great advantages, especially when combined with robotic technology. Case report: We report a case of leiomyoma of the middle esophagus with treated with enucleation using a robotic-assisted thoracoscopic technique. Intraoperative esophagoscopy and transillumination were useful for identifying the esophagus and developing a plan of safe extra mucosal dissection. Together with robotic assistance this seems to minimize intraoperative risks including that of mucosal injury while also improving postoperative recovery time.
\end{abstract}

Keywords

Esophageal leiomyoma, benign tumor, robotics, enucleation, minimally invasive surgery.

\section{INTRODUCCIÓN}

Todos los tumores estromales gastrointestinales tipo GIST (gastrointestinal stromal tumor) deben ser considerados como potencialmente malignos (1). Los tumores benignos del esófago son poco frecuentes y el leiomioma es el más común de los tumores benignos, representa más del $80 \%$ (2). Se originan de la capa circular muscular del esófago distal, esófago medio y, particularmente, en la unión gastroesofágica; las dimensiones que han sido reportadas en la literatura van desde $1 \mathrm{~cm}$ hasta los $29 \mathrm{~cm}$, y es el tipo de lesión ideal para cirugía de preservación de órgano. Los leiomiomas 
esofágicos han sido resecados convencionalmente mediante toracotomía abierta; sin embargo, han surgido técnicas toracoscópicas e inclusive el surgimiento de técnicas asistidas por robot que dan el beneficio de la imagen tridimensional (3D), que pueden disminuir el riesgo de perforación de la mucosa esofágica y mejorar la disección de las estructuras, que probablemente se convertirá en una ventaja frente a otros procedimientos quirúrgicos en un futuro (3). Se presenta el caso de una enucleación de leiomioma esofágico por robot con transiluminación intraoperatoria con esofagoscopia, que facilita la disección segura del plano extramucoso y logra la preservación de órgano.

\section{CASO CLÍNICO}

Se trata de una mujer de 41 años de edad que presentó antecedentes de disfagia desde el 2013. Una tomografía de tórax evidenció una lesión esofágica de 4,1 $\mathrm{cm}$ (Figuras 1A y 1B), a quien en 2013 intentan realizarle de modo extrainstitucional una resección por toracoscopia; sin embargo, se convierte en toracotomía por tumor de mediastino que compromete la pared del esófago torácico, se toman biopsias compatibles con fascículos de músculo liso sin pleomorfismo nuclear ni mitosis, la necrosis es compatible histopatológicamente con leiomioma benigno del esófago sin realización de inmunohistoquímica. En 2017, por persistencia de la disfagia, se realizaron estudios de extensión: un esofagograma con visualización de una lesión de $4,1 \mathrm{~cm}$ que produce alteración del tránsito esofágico (Figura 1C). Se confirma como masa submucosa por ultrasonografía endoscópica (Figura 1D) de aproximadamente $3,6 \mathrm{~cm}$ en su eje mayor a lo largo de la pared anterior del esófago en el tórax superior, inferior a la vena ácigos, apareciendo como masa hipo vascular sin adenopatías paraesofágicas.
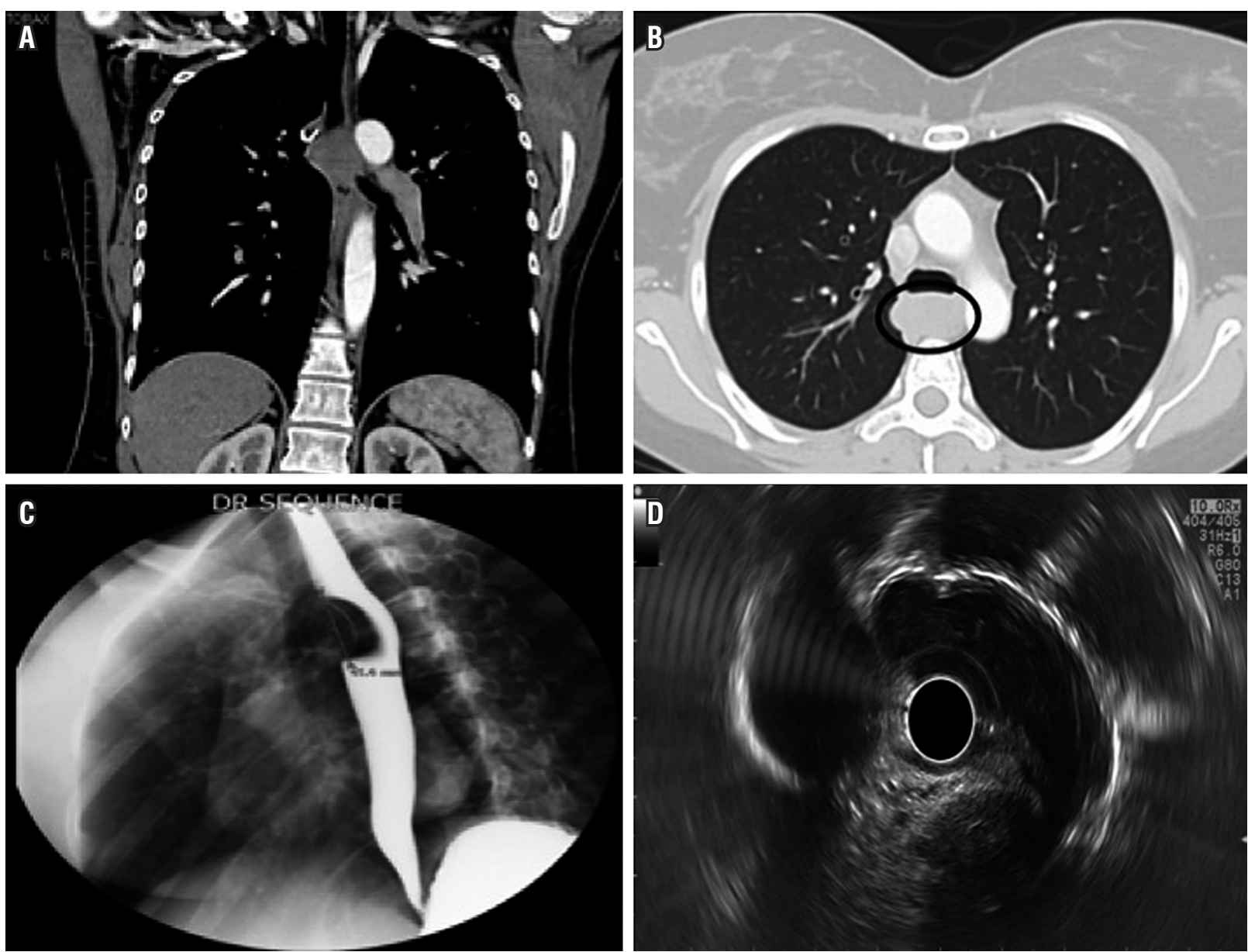

Figura 1. A y B. Tomografía de tórax con contraste: evidencia una masa esofágica heterogénea con medias de 4,1 centímetros. C. El esofagograma demuestra un defecto de llenado en el esófago con superficie mucosa lisa intacta. D. La ultrasonografía endoscópica del esófago revela una masa hipoecoica bien circunscrita originada en la capa muscular propia. 
La resección del tumor se consideró necesaria debido al tamaño de la lesión.

Bajo anestesia general utilizando un tubo de doble lumen en la posición de decúbito lateral izquierdo, se aisló el pulmón derecho y se colocaron trocares; un trocar de robot de $8 \mathrm{~mm}$ se colocó en la línea axilar media por encima de la quinta costilla y se colocó una cámara en este trocar mientras que otros puertos se colocaron bajo visualización directa. A continuación, se colocó un trocar de robot de $8 \mathrm{~mm}, 1 \mathrm{~cm}$ lateral a la columna vertebral sobre la octava costilla. Un tercer trocar de robot de $8 \mathrm{~mm}$ se colocó a $8 \mathrm{~cm}$ del último trocar sobre la octava costilla. Después se colocó un puerto de $12 \mathrm{~mm}$ a $8 \mathrm{~cm}$ del último trocar sobre la octava costilla cerca de la línea axilar posterior. Finalmente, se colocó un trocar de $12 \mathrm{~mm}$ a $8 \mathrm{~cm}$ inferior al puerto de la cámara sobre el diafragma. El robot fue entonces acoplado con brazos de robot conectados a los instrumentos de articulación tipo muñeca, que permiten una libertad de movimiento para el instrumental quirúrgico de más de 3 ejes. Se utilizaron pinzas grasper, disectores curvos, disectores con punta roma y pinzas porta yunque para la resección.

El tumor se identificó en el lado de la cabeza del arco ácigos a través de la pleura mediastínica (Figura 2A). La pleura mediastínica, la adventicia del esófago y la capa muscular propia se seccionaron longitudinalmente para exponer la cápsula tumoral (Figura 2B). El tumor parecía surgir principalmente de la lámina muscular de la mucosa, y un tumor redondo, elástico y duro fue enucleado con éxito por disección casi roma sin lesión de la mucosa (Figura 2C).
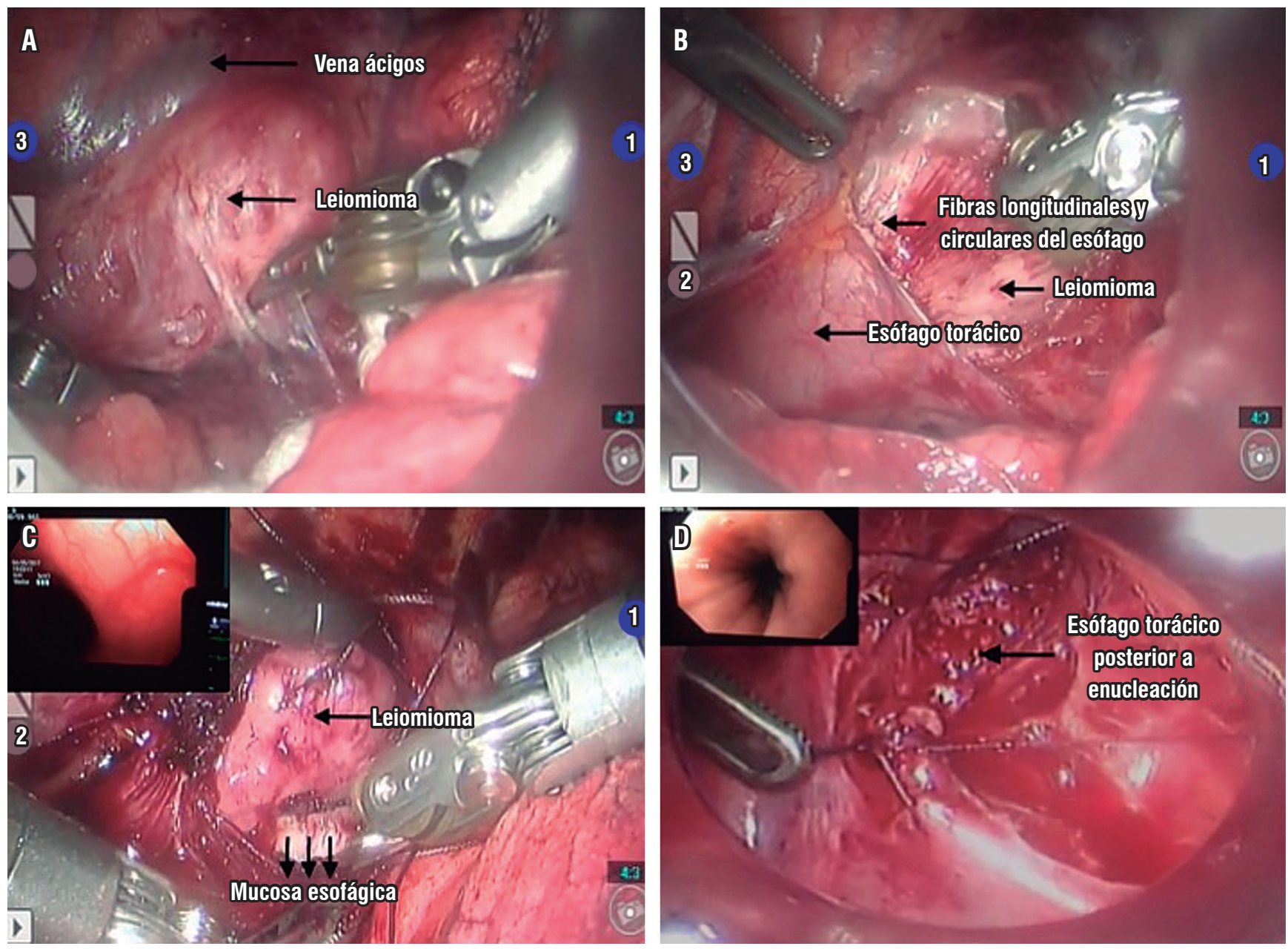

Figura 2. A. Imagen del leiomioma cubierto por pleura mediastínica del esófago antes de la disección. B y C. La visualización del leiomioma después de la disección con la inspección esofagoscópica durante la cirugía, asegurando la completa escisión del tumor y D. la integridad de la mucosa después de la enucleación con guía endoscópica. 
Después de la enucleación del tumor, la pleura mediastínica, la adventicia del esófago y la muscular propia del esófago se cerraron con suturas continuas (Figura 2D) y se colocó un tubo de tórax.

Se retiró el tubo de tórax el primer día postoperatorio, al día 1 postoperatorio se realizó un esofagograma que reveló mucosa esofágica lisa intacta sin fugas o estenosis (Figura 3A). El paciente inició la ingesta por vía oral a partir del día 1 del postoperatorio. El curso después de la cirugía fue favorable y se dio egreso a las 24 horas del postoperatorio. El examen histopatológico del espécimen resecado (Figuras 3B y C) mostró células tumorales en forma de huso, que parecían difusamente positivas para la desmina, pero negativas para el DOG-1, c-KIT, CD34 y S100 en el examen de inmunohistoquímica. El diagnóstico patológico final fue leiomioma del esófago sin evidencia de malignidad.

\section{DISCUSIÓN}

Todos los tumores estromales gastrointestinales tipo GIST deben ser considerados como potencialmente malignos (1), pero el leiomioma es el tumor mesenquimal benigno más común y representa más del $80 \%$ de los tumores esofágicos benignos. Puede ocurrir a cualquier edad, pero presenta un pico de incidencia entre la tercera y quinta década de la vida. Su localización usualmente se presenta en el tercio medio $y$ en el tercio inferior del esófago (2). Estos tumores son intramurales, tratándose de un tumor de crecimiento lento, y el tamaño suele ser $<5 \mathrm{~cm}$ (3). La mitad de los pacientes es asintomática y, en los pacientes sintomáticos, la disfagia y el malestar epigástrico son los síntomas más frecuentes (4); pero la regurgitación, el sangrado gastrointestinal alto y la pérdida de peso también pueden ocurrir (5).

Una vez el hallazgo se ha presentado, la esofagogastroscopia combinada con la ultrasonografía endoscópica del tumor es obligatoria para excluir el cáncer de esófago como diagnóstico diferencial. El aspecto típico de los GIST y los leiomiomas es de lesión homogénea e hipoecoica con un margen claro originadas en la musculatura propia o de la mucosa, que se pueden diferenciar de otras lesiones benignas del esófago como los lipomas hiperecoicos, y pueden ser fácilmente diferenciados (6-16); aunque algunos reportes identifican calcificaciones en el 1,8\% de los tumores, debido a que esta característica parece ser exclusiva de los leiomiomas esofágicos (7). Adicionalmente, La tomografía axial computarizada (TAC) da características de posible benignidad o malignidad, pero no excluye la posibilidad de malignidad ya que debe incluir la valoración histológica e inmunohistoquímica (8).

Se debe realizar una biopsia de la masa esofágica preoperatoriamente para distinguir entre GIST y leiomioma esofágico, aunque las guías de la National Comprehensive Cancer Network (NCCN) no sugieren biopsia preoperatoria de las masas resecables, debido a que los GIST pueden ser blandos y frágiles, y la biopsia puede causar hemorragia e incrementa el riesgo de diseminación del tumor por la ruptura de la pseudocápsula, e incluso la localización submucosa y la biopsia por aguja fina (FNA) pueden ser insuficientes para diferenciar entre el leiomioma y la variante maligna GIST (1). Sin embargo, las opciones de manejo se basan en el tamaño de las lesiones: para lesiones pequeñas $(<2 \mathrm{~cm})$ y asintomáticas, debe establecerse un consenso sobre la indicación de cirugía y el mejor método de tratamiento.
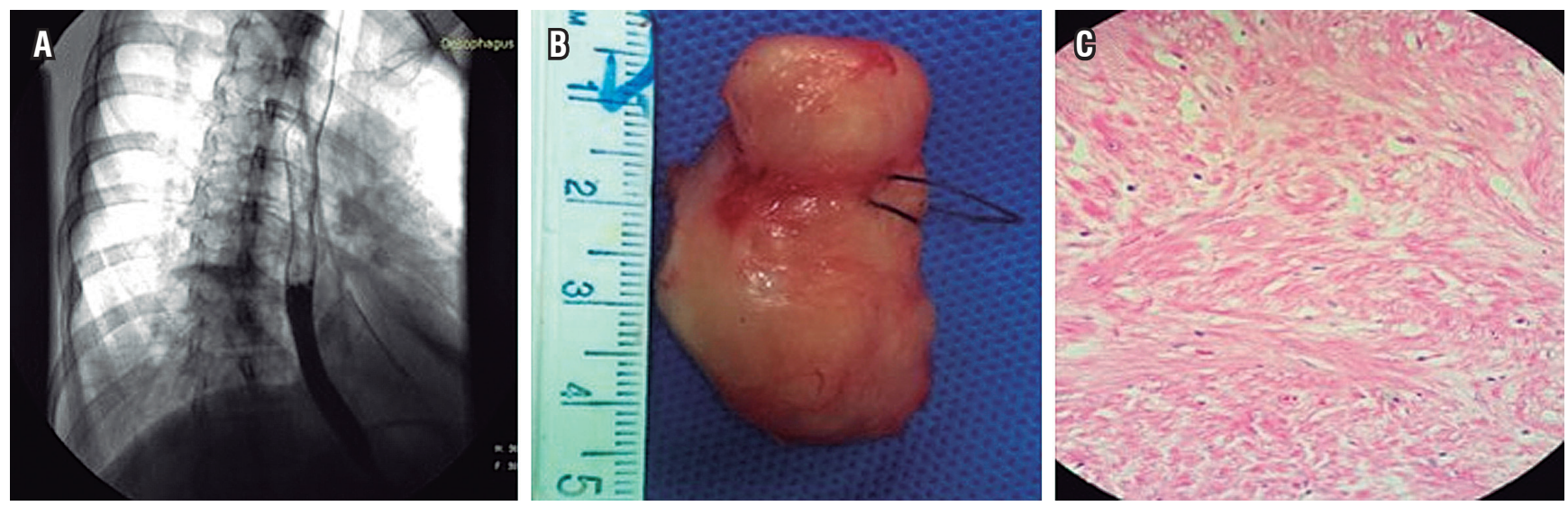

Figura 3. A. Esofagograma postoperatorio: evidencia de integridad del esófago torácico sin evidencia de estenosis y sin evidencia de fístulas. B. Pieza quirúrgica del leiomioma después de la enucleación que revela una masa de $4 \mathrm{~cm}$ con una pseudocápsula intacta. C. Vista microscópica del tumor (inserción H/E x 200). 
Algunos investigadores sugieren y recomiendan la evaluación por ecografía endoscópica cada 1 o 2 años. Las razones de este enfoque son las siguientes: el leiomioma es un tumor de crecimiento lento, la transformación maligna es extremadamente rara y el trauma quirúrgico puede ser más perjudicial para el paciente (9). Sin embargo, en las lesiones grandes $(>5 \mathrm{~cm})$ o sintomáticas, sin importar el tamaño, se debe resecar el tumor considerando la enucleación quirúrgica (10), como en el caso de nuestra paciente con una lesión de $4,1 \mathrm{~cm}$, pero sintomática. Las opciones de manejo del leiomioma esofágico incluyen enucleación por toracotomía, enucleación endoscópica, inyección de alcohol, enucleación por videotoracoscopia y enucleación asistida por robot; e incluso el surgimiento de la disección endoscópica submucosa tunelizada (ESTD) para la resección de tumores submucosos del esófago (11). Sin embargo, la elección del procedimiento de resección del tumor depende de las habilidades del operador debido a que no hay criterios formales para la selección del enfoque quirúrgico. Incluso, En casos de leiomiomatosis esofágica difusa o leiomioma esofágico gigante, a menudo se requiere esofaguectomía (6).

La enucleación esofágica del leiomioma vía abierta fue durante mucho tiempo el estándar de manejo de los tumores benignos, pero con la aparición del enfoque mínimamente invasivo se ha visto un descenso en el porcentaje de efectos adversos como el dolor y tiempo de estancia hospitalaria, en comparación con los procedimientos abiertos (7). Adicionalmente, estas técnicas mínimamente invasivas son más exigentes que la cirugía abierta debido a la visión bidimensional, la coordinación disminuida entre los ojos y las manos y la disminución de los grados de libertad al realizar los movimientos (5). Es por ello que los sistemas asistidos por robot también son de valor añadido en procedimientos mínimamente invasivos de resección toracoscópica del tumor esofágico, en su disección a lo largo de estructuras vitales como la vena pulmonar, vena ácigos, la aorta o la tráquea; que logra una visión tridimensional y mejora la coordinación de los ojos y las manos del cirujano, lo que podría llevar a reducir el riesgo de perforación de la mucosa esofágica (8).

La guía endoscópica intraoperatoria se ha convertido en una herramienta útil en la enucleación debido a que puede identificar la integridad de la mucosa, ya que la complicación postoperatoria más frecuente se da por la laceración de la mucosa. La evidencia de una mucosa íntegra permite un inicio más temprano de la vía oral, por lo cual algunos autores sugieren que la esofagoscopia intraoperatoria es esencial para el desempeño de la enucleación de tumores submucosos benignos del esófago. Proporciona un procedimiento seguro y preciso, y disminuye el riesgo de complicaciones postoperatorias (12-20). Por ello se decidió rea- lizar un abordaje asistido por robot con guía endoscópica intraoperatoria. Sin embargo, la desventaja de este procedimiento es que se requiere de la disponibilidad del equipo y de un médico bien entrenado.

El abordaje asistido por robot se ha asociado con una menor incidencia de lesión de la mucosa, debido a que la toracoscopia asistida robótica, particularmente en enucleación de tumor benigno esofágico, puede proporcionar una distinción más clara entre las capas anatómicas debido a la visión estereoscópica 3D ampliada superior, minimizando así las posibilidades de una perforación de la mucosa esofágica, en comparación con videotoracoscopia (VATS); y sobre el mediastino evitando la mortalidad quirúrgica, la pérdida de sangre intraoperatoria y reducción de la estancia hospitalaria debido a la disminución del dolor postoperatorio y de las fugas por laceración de la mucosa, y reincorporación más rápida a las actividades diarias $(14,18,19)$. Las ventajas se hacen más pronunciadas cuando la lesión está hacia el extremo del esófago torácico; es decir, el esófago torácico superior y hacia el hiato, donde se encuentran estructuras vitales $(13,14)$.

La cirugía esofágica asistida por robot está entrando en un campo de revolución, pero aún faltan estudios y evidencia que soporten un enfoque robótico en comparación con otros enfoques; por supuesto, los tiempos quirúrgicos prolongados y los altos costos que requiere este abordaje quirúrgico son algunos de los obstáculos para una amplia aceptación de esta técnica. Se requieren investigaciones adicionales en el campo de la enucleación de lesiones esofágicas por robot para indicar esta técnica como primera línea de abordaje en comparación con otras técnicas mínimamente invasivas (14-18). En nuestro caso se ofreció al paciente un abordaje asistido por robot debido a la ubicación de la lesión y el antecedente quirúrgico, sin complicaciones intraoperatorias ni postoperatorias.

La distinción preoperatoria de leiomioma y el GIST son difíciles de realizar en lo que hemos mencionado. Por tanto, la evaluación de la pieza quirúrgica postoperatoria con hematoxilina eosina y la inmunohistoquímica postoperatoria es el patrón de oro para el diagnóstico de cualquier leiomioma, debido a que estas lesiones son CD34 y CD117 negativo, sin mutaciones c-KIT y son positivos para la desmina y la actina de músculo liso (SMA); mientras que los GIST suelen ser (aunque no siempre) negativos (6-17). Los GIST presentan un patrón de inmunohistoquímica diferente, alrededor del $95 \%$ de los GIST es positivo para c-KIT (CD117), 60\%-70\% para CD34, 30\%-40\% para SMA y 5\% para la proteína S-100 (1-15).

Para terminar, el principal método de seguimiento de los pacientes posterior a la enucleación de tumores benignos del esófago aún no ha sido establecida. En algunos casos de GIST, el seguimiento regular ha sido recomendado con 
base en la probabilidad de recurrencia de la lesión por los hallazgos de la pieza quirúrgica; pero a diferencia del GIST, la recurrencia de otros tipos de tumores esofágicos como el leiomioma son muy raros, por lo que puede no ser necesario aplicar seguimiento oncológico para estos tumores, sino un seguimiento clínico periódico (11-21).

\section{CONCLUSIONES}

La enucleación de leiomioma esofágico con asistencia robótica no solo permite la resección completa de la lesión, además ofrece ventajas como mejor exposición de las estructuras, una visión en 3D, magnificación de la imagen y mejor coordinación de los ojos y las manos del cirujano; sin embargo, faltan estudios sobre el abordaje por videotoracoscopia. Aunque se ha establecido que este enfoque es factible y eficaz en el tratamiento quirúrgico de los leiomiomas del esófago.

\section{Aprobación ética}

Se obtuvo el consentimiento informado por escrito del paciente para la publicación de este documento.

\section{REFERENCIAS}

1. Jiang P, Jiao Z, Han B, Zhang X, Sun X, Su J, et al. Clinical characteristics and surgical treatment of oesophageal gastrointestinal stromal tumours. Eur J Cardiothorac Surg. 2010;38(2):223-7. doi: 10.1016/j.ejcts.2010.01.040.

2. Kernstine $\mathrm{KH}$, Andersen ES, Falabella A, Ramirez NA, Anderson CA, Beblawi I. Robotic fourth-arm enucleation of an esophageal leiomyoma and review of literature. Innovations (Phila). 2009;4(6):354-7. doi: 10.1097/ IMI.0b013e3181c46218.

3. DeUgarte DA, Teitelbaum D, Hirschl RB, Geiger JD. Robotic extirpation of complex massive esophageal leiomyoma. J Laparoendosc Adv Surg Tech A. 2008;18(2):286-9. doi: 10.1089/lap.2007.0067.

4. Choi SH, Kim YT, Han KN, Ra YJ, Kang CH, Sung SW, et al. Surgical management of the esophageal leiomyoma: lessons from a retrospective review. Dis Esophagus. 2011;24(5):3259. doi: 10.1111/j.1442-2050.2010.01144.x.

5. Iscan Y, Tunca F, Senyurek YG, Tezelman S, Terzioglu T. Thoracoscopic enucleation of a giant leiomyoma of the esophagus. Surg Laparosc Endosc Percutan Tech. 2013;23(1):e32-4. doi: 10.1097/SLE.0b013e318275b2a3.

6. Markakis CG, Spartalis ED, Liarmakopoulos E, Kavoura EG, Tomos P. Esophageal gastrointestinal stromal tumor: diagnostic complexity and management pitfalls. Case Rep Surg. 2013;2013:968394. doi: 10.1155/2013/968394.

7. Macke RA, Luketich JD, Nason KS, Schuchert MJ. Minimally invasive enucleation of a large, extensively cal- cified esophageal leiomyoma. J Thorac Cardiovasc Surg. 2014;147(4):e52-4. doi: 10.1016/j.jtcvs.2013.12.054.

8. Elli E, Espat NJ, Berger R, Jacobsen G, Knoblock L, Horgan S. Robotic-assisted thoracoscopic resection of esophageal leiomyoma. Surg Endosc. 2004;18:713-6. doi: 10.1007/ s00464-003-8829-y.

9. Luh SP, Hou SM, Fang CC, Chen CY. Video-thoracoscopic enucleation of esophageal leiomyoma. World J Surg Oncol. 2012;10:52. doi: 10.1186/1477-7819-10-52.

10. Khalaileh A, Savetsky I, Adileh M, Elazary R, Abu-Gazala M, Abu Gazala S, et al. Robotic-assisted enucleation of a large lower esophageal leiomyoma and review of literature. Int J Med Robot. 2013;9(3):253-7. doi: 10.1002/rcs.1484.

11. Li QY, Meng Y, Xu YY, Zhang Q, Cai JQ, Zheng HX, et al. Comparison of endoscopic submucosal tunneling dissection and thoracoscopic enucleation for the treatment of esophageal submucosal tumors. Gastrointest Endosc. 2017;86(3):485-491. doi: 10.1016/j.gie.2016.11.023.

12. Shin S, Choi YS, Shim YM, Kim HK, Kim K, Kim J. Enucleation of esophageal submucosal tumors: a single institution's experience. Ann Thorac Surg. 2014;97(2):4549. doi: 10.1016/j.athoracsur.2013.10.030.

13. Chen X, Xi Y, Wang H, Tan L. Minimally invasive surgery for giant esophageal leiomyoma: a case report \& review of the literatures. J Thorac Dis. 2017;9(1):E26-E31. doi: 10.21037/jtd.2017.01.34.

14. Kumar A, Asaf BB. Robotic thoracic surgery: The state of the art. J Minim Access Surg. 2015;11(1):60-7. doi: 10.4103/0972-9941.147693.

15. Cho SY, Moon HJ, Kim JW, Cho SK, Kim BG, Joo SK, et al. Clinicopathologic feature of esophageal submucosal tumors treated by surgical approach]. Korean J Gastroenterol. 2013;61(2):71-4. doi: 10.4166/kjg.2013.61.2.71.

16. How CH, Lee JM. The robotic approach for enucleation of a giant esophageal lipoma. J Robot Surg. 2017;11(2):267269. doi: 10.1007/s11701-016-0668-7.

17. Robb WB, Bruyere E, Amielh D, Vinatier E, Mabrut JY, Perniceni T, et al. Esophageal gastrointestinal stromal tumor: is tumoral enucleation a viable therapeutic option? Ann Surg. 2015;261(1):117-24. doi: 10.1097/ SLA.0000000000000505.

18. Kocher GJ. Robotic-assisted thoracoscopic surgery: state of the art and future perspectives. J Thorac Dis. 2017;9(7):1855-7. doi: 10.21037/jtd.2017.06.139.

19. Compean S, Gaur P, Kim M. Robot assisted thoracoscopic resection of giant esophageal leiomyoma. Int J Surg Case Rep. 2014;5(12):1132-4. doi: 10.1016/j.ijscr.2014.11.003.

20. Jeon HW, Choi MG, Lim CH, Park JK, Sung SW. Intraoperative esophagoscopy provides accuracy and safety in video-assisted thoracoscopic enucleation of benign esophageal submucosal tumors. Dis Esophagus. 2015;28(5):437-41. doi: 10.1111/dote. 12220 .

21. Ben-David K, Alvarez J, Rossidis G, Desart K, Caranasos T, Hochwald S. Thoracoscopic and Laparoscopic Enucleation of Esophageal Leiomyomas. J Gastrointest Surg. 2015;19(7):1350-4. doi: 10.1007/s11605-015-2817-0. 